\title{
Long-Term Administration of Anthraquinone Rhein on Induction of Constipation in Sprague-Dawley Rats via SCF/c-Kit Signaling Pathways
}

\author{
Bo Zhang $\mathbb{D}^{1},{ }^{1}$ Mingdong Huo $\mathbb{D}^{1},{ }^{1}$ Zhicheng Chen $\mathbb{D}^{1},{ }^{1}$ Feng Gao $\mathbb{D}^{2},{ }^{2}$ Yan Liu $\left(\mathbb{D},{ }^{1}\right.$ \\ and Xingxin Zhou $\mathbb{D}^{1}$ \\ ${ }^{1}$ Department of Anal and Intestinal Diseases, ZhongDa Hospital, Southeast University, Nanjing 210009, China \\ ${ }^{2}$ Department of Laboratory Medicine of Medical School, Southeast University, Nanjing 210009, China \\ Correspondence should be addressed to Mingdong Huo; huomingdong2018@126.com
}

Received 26 October 2020; Revised 28 March 2021; Accepted 15 April 2021; Published 26 April 2021

Academic Editor: Rafał Filip

Copyright (c) 2021 Bo Zhang et al. This is an open access article distributed under the Creative Commons Attribution License, which permits unrestricted use, distribution, and reproduction in any medium, provided the original work is properly cited.

\begin{abstract}
Background and Study Aims. It has been shown that abuse of laxatives is becoming a serious problem; therefore, a comprehensive understanding of its effect and possible mechanism on colon motility is essential to select effective treatments and avoid their abuse. Herein, we aimed to investigate the long-term stimulation of rhein on induction of constipation in rats and its underlying mechanisms. Materials and Methods. After establishing rat models of constipation, the rats were randomly divided into two equal subgroups and administered daily with normal saline (model control group) or $10 \mathrm{ml} / \mathrm{kg}$ PEG4,000 (PEG-treated group). Simultaneously, normal Sprague-Dawley (SD) rats were administered with normal saline (normal group). Physiological and fecal parameters were calculated, and intestinal transmission function was evaluated. After scarification, colonic tissues were freshly prepared for histological localization detected by immunohistochemical analysis and for the expression of stem cell factor (SCF) and c-kit proteins determined by western blot assay. Results. Following the initiation of rhein-induced rat constipation, body weight was lost slightly, the first time of black stool discharge was obviously longer, and the fecal moisture and number of fecal pellets decreased distinctly as compared with normal group. A decreased expression of SCF and c-kit was detected in model control group in comparison with normal group. Notably, compared with model control group, neither the alterations of fecal parameters and intestinal transmission function were effectively restored, nor the expression of SCF and c-kit was markedly elevated after administration of PEG4,000 for $30 \mathrm{~d}$. Conclusion. Long-term stimulation of rhein can develop the constipation via $\mathrm{SCF} / \mathrm{c}$-kit signaling pathway, yet the symptoms of constipation and colon power cannot be alleviated or restored by PEG4,000. Collectively, these findings strongly suggest that long-term use of anthraquinone laxatives should be avoided for clinical treatment of constipation.
\end{abstract}

\section{Introduction}

Constipation is often a chronic functional gastrointestinal disorder characterized by symptoms such as infrequent bowel movements, difficulty during defecation, and sensation of incomplete bowel evacuation, which affect people of all ages and both sexes [1], and the prevalence of constipation ranged from $2 \%$ to $34 \%$ [2]. With the rhythm of social work and the pace of life speeding up, the incidence of people's constipation is increasing and an increasing number of people are suffering from it, consequently leading to a significant personal and public health burden [3]. Current straightway treatment strategies for constipation mainly focus on modulating motility of gastrointestinal tract. Although Western medicines have been the dominant treatment for constipation patients, their adverse effects and part of patients' intolerance to pharmacotherapy make traditional Chinese medicine one of their alternatives [4]. Rhein, a free anthraquinone compound isolated from rhubarb, has been serving as a mild laxative agent as well as an astringent in the Chinese population since ancient times [5]. More recently, it has been reported that abuse of 
laxatives, including misuse in treatment of obesity and persistent use in treatment of chronic constipation, is becoming a serious problem for relatively poor understanding of molecular aetiology [6]; therefore, a comprehensive understanding of the effect and possible mechanism of anthraquinone laxative on colon motility is essential to select effective treatments and avoid their abuse.

Up to now, compelling evidence has revealed that constipation is related to pathologic alteration in interstitial cells of Cajal (ICCs), receptor tyrosine kinase c-kit, and its ligand-stem cell factor (SCF) system as well as abnormality of the enteric nervous system $[7,8]$, whereas the pathogenesis of constipation is still not very clear. Of note, the decreased numbers of ICCs and defects in its networks affect gut motility, leading to diarrhea and/or constipation [9], yet whether the SCF/c-kit signaling pathway is associated with rhein-induced constipation remains to elucidate.

Polyethylene glycol (PEG), a nonabsorbable and nonmetabolizable polymer, has been reported to be one of the most widely prescribed laxative agents as the first-line treatment of functional constipation in short- and longterm, as well as for prevention and resolution of fecal impaction in adult and pediatric populations [10]. Recently, several practice guidelines have been recommended to use PEG-based laxatives as the first-line pharmacological treatment for chronic constipation $[11,12]$. Herein, we aimed to investigate the role of SCF/ckit signaling pathway in rhein-induced constipation and further to elucidate whether PEG4,000 can restore colon power and alleviate symptoms of constipation in the rhein-induced model.

\section{Materials and Methods}

2.1. Chemicals. Rhein,4,5-Dihydroxy-9,10-dioxo-9,10-dihydro-2-anthracenecarboxylic acid $\left(\mathrm{C}_{15} \mathrm{H}_{8} \mathrm{O}_{6}\right)$, was purchased from Nanjing Zelang Medical Technology Co. Ltd. (Nanjing, China) and dissolved in normal saline at the concentration of $2 \%$. Polyethylene glycol (PEG) 4,000 was purchased from Bofu-Yipusheng Pharmaceutical Co. (Tianjin, France). Hematoxylin and eosin (H\&E) staining kit was purchased from Sigma-Aldrich (St. Louis, MO, USA). BCA protein assay kit was obtained from Biyuntian biotechnology co. Ltd. (Beijing, China). Anti-c-kit (No. ab32363), SCF (No. ab64677), and $\beta$-actin antibodies were obtained from Abcam Shanghai Trading Co., Ltd. (Shanghai, China), and secondary horseradish peroxidase-conjugated goat anti-rabbit was obtained from Abcam Shanghai Trading Co., Ltd. (Shanghai, China).

2.2. Experimental Animals. Adult Sprague-Dawley rats weighting 180 195 g were provided by the medical experimental animal center of Southeast University. Rats were maintained under constant environmental conditions with a $12 \mathrm{~h} \mathrm{light} / \mathrm{dark}$ cycle at a temperature of $23 \pm 2^{\circ} \mathrm{C}$ and a relative humidity of $50 \pm 10 \%$, and given free access to a standard laboratory chow and water. All rats were acclimatized to the facilities for a week prior to experiments. The handling of animals and the experimental procedures were in accordance with the institutional animal guidelines and the Ethics Committee of the Zhongda Hospital, Medical School of Southeast University (20171209007), Nanjing, China.

2.3. Experimental Design. After adaptation for a week, 36 healthy rats were randomly assigned into two main groups, Group1 (received normal saline) and Group 2 (received three phases of gavage with $2 \%$ rhein to develop the symptoms of constipation). In brief, the rats in Group 2 received gavage with $2 \%$ rhein $(1.2 \mathrm{ml} / \mathrm{kg})$ once every other day in the first phase (P1) until disappearance of loose stools for $80 \%$ rats. Subsequently, gavage was repeated daily for consecutive $5 \mathrm{~d}$ and then stopped for $2 \mathrm{~d}$ in the second phase (P2) until disappearance of loose stools for $80 \%$ rats. Thereafter, the rats received gavage with $2 \%$ rhein $(1.6 \mathrm{ml} / \mathrm{kg})$ for consecutive $5 \mathrm{~d}$ and then stopped for $2 \mathrm{~d}$ in the third phase (P3) until appearance of dry and hard feces (Figure 1), during which the frequency of defecation, fecal moisture, and first time of black stool discharge were taken as markers of model establishment.

The constipated rats were further equally subdivided into two subgroups and administered daily by gavage with either normal saline $(10 \mathrm{ml} / \mathrm{kg}$ ) (model control group) or PEG4,000 aqueous solutions $(10 \mathrm{ml} / \mathrm{kg}$ ) (PEG-treated group) for consecutive $30 \mathrm{~d}$. Similarly, equivalent volume of saline vehicle was given to the rats in normal group (Figure 1). During the period, body weight, an important marker for constipation in rat, was detected every other day to adjust the dose of PEG4,000 and normal saline. At the end of experiments, the colonic tissues were prepared freshly from the sacrificed rats for immunohistochemistry and western blot assay, respectively.

2.4. Measurement of Fecal Parameters. Each mouse was put into a clean, empty, bedding-free cage to collect fresh fecal pellets and the frequency of defecation was counted at $0-8 \mathrm{~h}$ period. Fresh feces (A) were weighted immediately using an electric balance, and their dry weight (B) was measured after drying at $150^{\circ} \mathrm{C}$ for $15 \mathrm{~min}$. Then, the fecal moisture was calculated according to the following equation: the fecal moisture $(\%)=(\mathrm{A}-\mathrm{B}) / \mathrm{A} \times 100$.

2.5. Intestinal Transmission Function. A biological staining method with intragastric feeding of activated carbon was used to reflect the intestinal transmission function according to the first time of black stool discharge [13]. Briefly, except for water ad libitum, all rats were fasted to empty the intestines and then orally administered with $3 \mathrm{ml} 10 \%$ activated carbon suspension. Thereafter, the transit time was calculated from the beginning of intragastric feeding to the first time of black stool discharge.

2.6. Histology. Upon scarification, the colonic tissues were fixed in $10 \%$ buffered formalin for $24 \mathrm{~h}$, dehydrated through graded alcohol and embedded in paraffin, and serially cut 


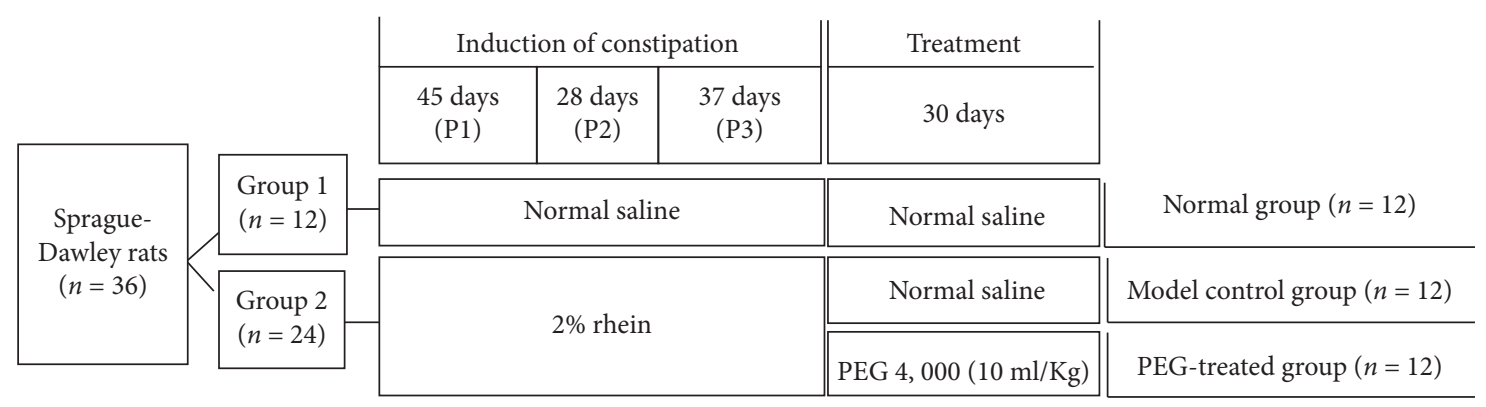

FIgURE 1: Experimental protocol. P1, the first phase; P2, the second phase; P3, the third phase. PEG, polyethylene glycol.

into cross sections of $5 \mu \mathrm{m}$ thickness. Then, the sections were stained with $\mathrm{H} \& \mathrm{E}$ according to standard procedures. Thereafter, histopathologic features were observed under a light microscope (Olympus BX51, Japan) equipped with a color CCD camera (Media Cybernetics Evolution MP 5.0).

2.7. Immunohistochemistry. $5 \mu \mathrm{m}$ sections were cut from paraffin blocks and incubated with primary monoclonal anti-SCF or anti-c-kit $(1: 1000)$ at $4^{\circ} \mathrm{C}$ overnight. Then, the sections were re-incubated with a secondary anti-rabbit biotinylated antibody $(1: 2000)$ for $1 \mathrm{~h}$ at room temperature after washing. Thereafter, the stained slides were examined to identify cellular localization of SCF and its receptor c-kit under a light microscope (Olympus BX51, Japan) equipped with a color CCD camera (Media Cybernetics Evolution MP $5.0)$.

2.8. Western Blot. Total protein was extracted from colonic tissues homogenized in iced phosphate-buffered saline containing protease inhibitors. After centrifugation, the concentration of total protein in supernatants was determined by BCA protein assay kit. Equal amounts of protein $(40 \mu \mathrm{g})$ were loaded on each lane of $10 \%$ SDS-polyacrylamide gel electrophoresis, and the separated proteins were transferred to nitrocellulose membranes (Bio-rad) with a semi-dry transfer. Subsequently, the membranes were blocked with 5\% non-fat dry milk for $1 \mathrm{~h}$ at room temperature and then incubated separately with primary antiSCF or anti-c-kit $(1: 1000)$ overnight at $4^{\circ} \mathrm{C}$. After washing, the membranes were incubated with horseradish peroxidase-conjugated goat anti-rabbit secondary antibody (1: 2,000 ) at room temperature for $1 \mathrm{~h}$. Thereafter, the blots were visualized using an enhanced chemiluminescence system (GE Healthcare, Chalfont, UK) and normalized to the expression of $\beta$-actin.

2.9. Statistical Analysis. Data are expressed as mean\pm standard deviation for experiments performed in triplicate. Statistical analysis was conducted using Statistical Package for Social Sciences (SPSS version 18.0; SPSS, Inc., Chicago, IL, USA). Continuous variables between two groups were compared using $t$-test, and comparison among groups was performed using one-way analysis of variance with Duncan's multiple range test. $P<0.05$ was considered to be significant difference.

\section{Results}

3.1. Body Weight and General Condition of Rats. Compared with initial body weight of rats, the corresponding final body weight increased significantly in the normal group $(P=0.0001)$, model control group $(P=0.0001)$, and PEG-treated group $(P=0.0001)$ (Table 1$)$. After administration for consecutive $30 \mathrm{~d}$, the final body weight in PEG-treated group was higher than that of model control $(P=0.0001)$. Additionally, the mean daily food intake $(P=0.292)$ and water consumption $(P=0.501)$ in rats with constipation were maintained at constant levels as compared with normal group (Table 1). Notably, no diarrhea was observed in either group during the treatment of constipation.

3.2. Effect of Rhein on Induction of Constipation. To investigate whether administration of rhein could induce constipation in SD rats, alterations in the excretion parameters, including frequency of defecation (the number of fecal pellets per day) and fecal moisture, were calculated. Owing to more feces remaining in colon for too long, the number of fecal pellets in model control group was less than that in normal rats $(P=0.0001)$, and the frequency of defecation in PEG-treated group was slightly higher than that in the model control group; however, the data failed to reach a statistical significance $(P=0.207)$ (Table 2$)$. Similarly, fecal moisture in constipated rats decreased distinctly compared with normal rats $(P=0.0001)$, whereas there was no significant difference between model control group and PEG-treated group $(P=0.463)$. The present results indicate that SD rat constipation model was successfully established, the symptoms of which cannot be alleviated by administration of PEG4,000.

3.3. Effect of Rhein on Intestinal Transmission Function. After gavage with activated carbon, the first time of black stool discharge was longer in the constipated rats as compared with normal rats $(P=0.0001)$. Although PEG4,000 accelerated the discharge of the first black stool, there was no significant difference between PEG-treated group and model control group $(P=0.246)$ (Figure 2$)$. All these results suggest that long-term stimulation of rhein on gastrointestinal can reduce colon power in the constipation model rats, and the 
TABLE 1: Effects on body weight, food intake, and water consumption of rats with or without constipation for $30 \mathrm{~d}(\bar{x} \pm s)$.

\begin{tabular}{|c|c|c|c|c|}
\hline \multirow{2}{*}{ Groups } & \multicolumn{2}{|c|}{ Body weight (g/rat) } & \multirow{2}{*}{ Daily food intake (g/rat) } & \multirow{2}{*}{ Daily water consumption (ml/rat) } \\
\hline & Initial & Final & & \\
\hline Normal group $(n=12)$ & $191.4 \pm 2.2$ & $412.8 \pm 3.4^{*}$ & $24.8 \pm 1.6$ & $70.8 \pm 3.5$ \\
\hline Model control group $(n=12)$ & $192.3 \pm 3.1$ & $394.3 \pm 4.5^{*}$ & $24.7 \pm 1.7$ & $68.6 \pm 3.2$ \\
\hline PEG-treated group $(n=12)$ & $191.8 \pm 1.9$ & $406.8 \pm 5.2^{*, \&}$ & $24.5 \pm 1.8^{\#}$ & $69.1 \pm 2.9^{\#}$ \\
\hline
\end{tabular}

Results are expressed as the mean \pm standard deviation. ${ }^{*} P<0.05$, compared with initial corresponding group; ${ }^{\&} P<0.05$, compared with model control group; ${ }^{\#} P>0.05$, compared with normal group and model control group. PEG, polyethylene glycol.

TABle 2: Alteration of stool in rhein-induced constipation of rats after administration with or without PEG4,000 for $30 \mathrm{~d}(\%)(\bar{x} \pm s)$.

\begin{tabular}{lcc}
\hline Groups & Frequency of defecation (number/rat) & Fecal moisture (\%) \\
\hline Normal group $(n=12)$ & $32.4 \pm 5.6$ & $56.88 \pm 3.62$ \\
Model control group $(n=12)$ & $18.2 \pm 4.8^{*}$ & $47.81 \pm 3.98^{*}$ \\
PEG-treated group $(n=12)$ & $20.6 \pm 4.2^{*}, \#$ & $49.20 \pm 3.27^{*}, \#$ \\
\hline
\end{tabular}

Results are expressed as the mean \pm standard deviation. ${ }^{*} P<0.05$, compared with normal group; ${ }^{\#} P>0.05$, compared with model control group. PEG, polyethylene glycol.

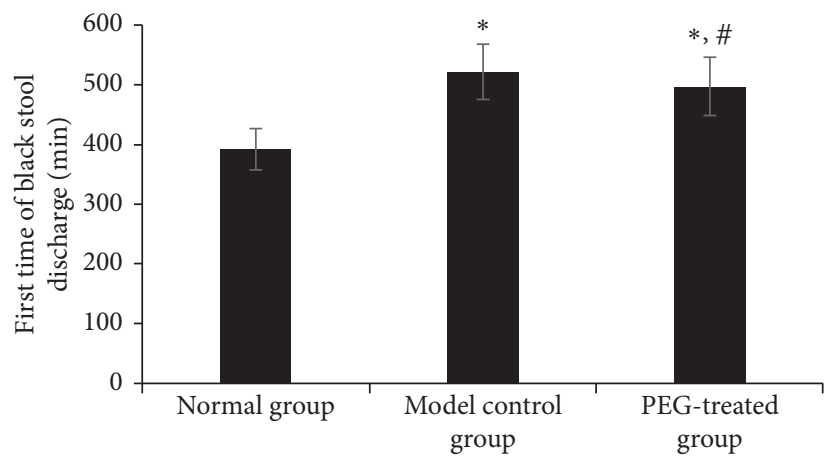

Figure 2: Comparing the first time of black stool discharge in rats after administration with or without PEG4,000 for $30 \mathrm{~d}$. Results are expressed as the mean \pm standard deviation, $n=12 .{ }^{*} P<0.05$, compared with normal group; ${ }^{\#} P>0.05$, compared with model control group. PEG, polyethylene glycol.

decrease of colon power cannot be improved by administration of PEG 4,000.

\subsection{Effects on Histological Alterations of Colonic Tissues.} After administration for $30 \mathrm{~d}$, the rats in normal group were just administered normal saline and showed no alteration of colon appearance. Of note, pathological changes including flatulence and dilation of colon, accumulation of gas and fluid in intestinal cavity, and the thinning of intestinal wall of colon were observed in model control group. After staining with $\mathrm{H} \& \mathrm{E}$, colonic sections showed an intact intestinal structure without any histological alterations in normal group (Figures 3(a) and 3(d)). Of note, chronic mucosal inflammation and vacuolar degeneration of myenteric plexus nerve cells were observed in model control group (Figures 3(b) and 3(e)), and a similar trend of alteration was observed in PEG-treated group (Figures 3(c) and 3(f)). All these results suggest that administration of PEG4,000 cannot significantly restore abnormal structure of colon in constipated model rats.

3.5. Location of SCF and c-Kit in Colonic Tissues of SD Rats. Numerous SCF positive cells were observed in all intestinal wall layers and deep brown granules appeared at the full- thickness of knot bowel wall in normal group (Figure 4(a)), whereas expression of SCF decreased significantly and only mild expression was shown in model control group (Figure 4(b)). Similarly, expression of c-kit was also observed in entire colon muscle layer, especially in circular muscular layer and intramuscular plexus area, and deep brown granule could be seen on ICCs in normal group (Figure 4(d)), while those in intramuscular plexus and ICCs appeared to be pale dyeing in model control group (Figure 4(e)), suggesting the expression of SCF and c-kit decreased significantly in model constipation rats compared with normal ones. Of note, a similar trend of alteration in colonic tissues was observed in PEG-treated group, and there was no significant difference as compared with model control group (Figures 4(c) and 4(f)).

3.6. Expression Level of SCF and c-Kit Proteins in Colonic Tissues of SD Rats. After administration for $30 \mathrm{~d}$, the expression level of SCF and c-kit proteins decreased significantly in model control group as compared with normal group, and there was a significant difference between them $(P=0.0001)$. Additionally, the expression level of SCF and c-kit proteins decreased slightly in constipated model rats administered with PEG4,000 in comparison with those with 


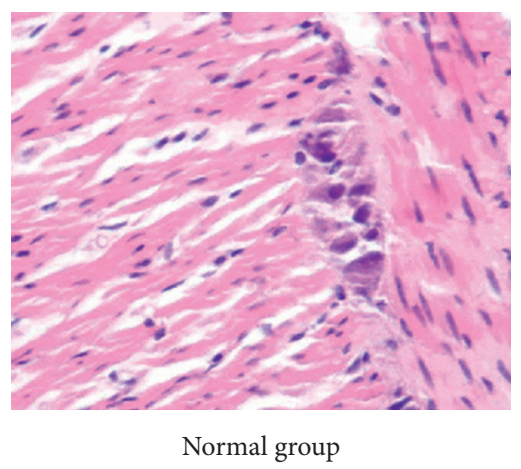

(a)

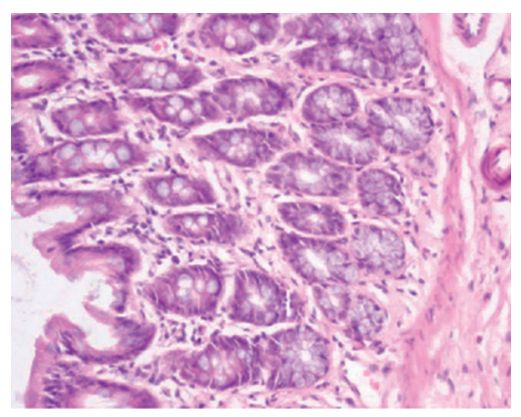

Normal group

(d)

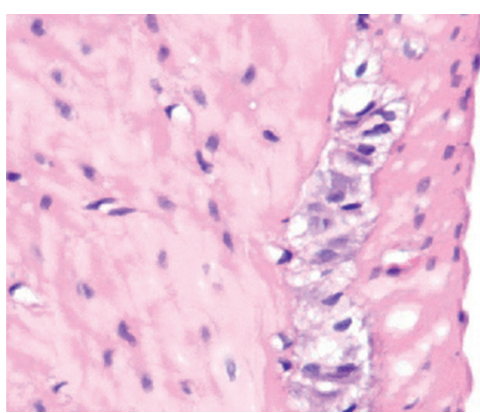

Model control group

(b)

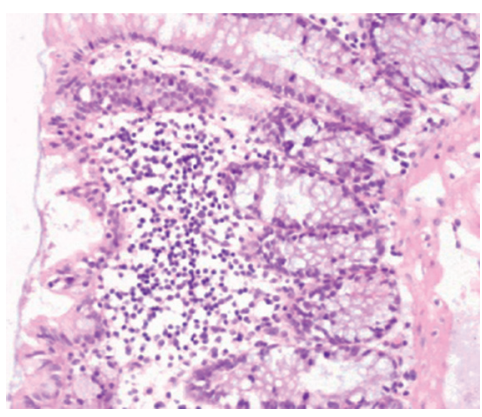

Model control group

(e)

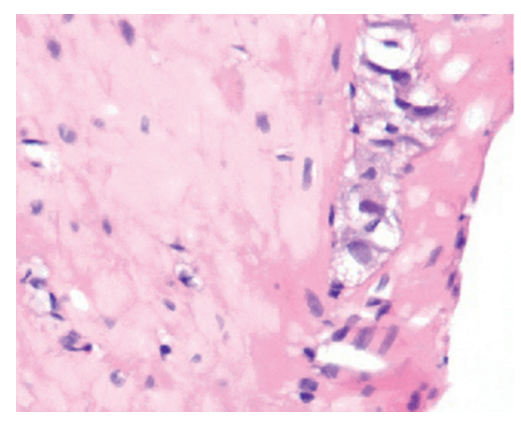

PEG-treated group

(c)

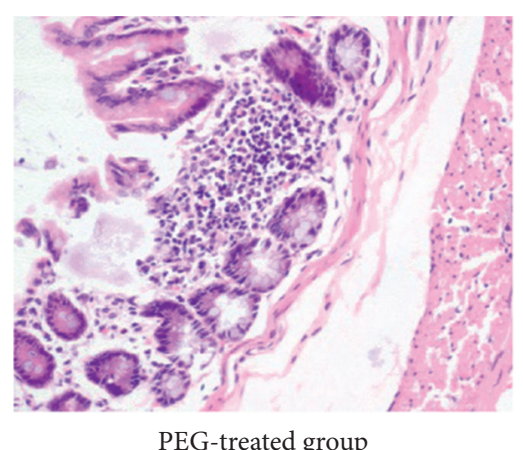

(f)

FIGURE 3: Histological sections of ganglia $(\mathrm{a}-\mathrm{c})$ and mucosa $(\mathrm{d}-\mathrm{f})$ morphology stained with hematoxylin and eosin in the colonic tissues of rats (400x). PEG, polyethylene glycol.

normal saline, and there was no significant difference between them $(P=0.081)$ (Figure 5), suggesting modeling can reduce the number of ICCs in colonic tissues, which cannot be effectively restored by administration of PEG4,000.

\section{Discussion}

Constipation most commonly occurs when the formed stool moves too slowly as it passes through the digestive tract. It not only causes a serious effect on the quality of life, but also induces or aggravates other diseases in patients [14]. A previous study reported that onset of some patients with constipation-predominant irritable bowel syndrome resulted from long-term use of Chinese and Western medicines [15]. Additionally, clinical observations also find that patients with slow transit constipation have the medical history of long-term use of anthraquinone laxatives [16]. Given the paucity of available clinical data, animal models are required to study the pathogeny of constipation and develop strategies for prevention and treatment [17]. Although the activated carbon-induced constipation in mice has been used to demonstrate the effects of drugs for constipation treatment [18], a megadose of activated carbon attaching to the mucosal surfaces of gastrointestinal tract results in the digestive tract obstruction [19]. Therefore, there is a need to develop optimized models to study clinical treatment of constipation.

Although there are great efforts in identifying the best treatment options for patients affected by chronic constipation, the results of constipation treatment are often still partial and unsatisfactory [20]. Recently, there is a growing consumer interest in herbal plants and medicinal foods that can either promote the state of well-being and/or be used as novel therapeutic drugs to treat constipation and its related diseases [21, 22]. Of note, rhein has been reported to exhibit linear pharmacokinetics between 50 and $200 \mathrm{mg}$ [23] and possess diverse pharmacological actions [24-27]. Some studies have also revealed the mechanisms of rhein on anti-inflammation [26], anti-oxidant [27], and others; until recently, the effect of rhein on the induction of constipation and its possible mechanism have not been fully addressed.

As we know, there are many potential causes of constipation that also affect the colon [28], among which laxatives can help relieve constipation and promote regular bowel movements; however, a previous study showed that overuse of laxatives is associated with a series of serious adverse effects [29]. In current study, as expected, the human-like symptoms of constipation were observed in SD rats by three phases of oral gavage with $2 \%$ rhein (Figure 1), which was demonstrated by the decreased fecal moisture and number of fecal pellets as well as the prolonged stool discharge. Using two models of experimental constipated mice, it was also found that a primary mechanism for slow transit constipation is a failure of peristalsis to propel colonic luminal contents through the colon, resulting in more time for bacterial degradation of stool solids and more time for salt and water absorption, consequently reducing stool frequency and stool weight dramatically [30]. All these results 


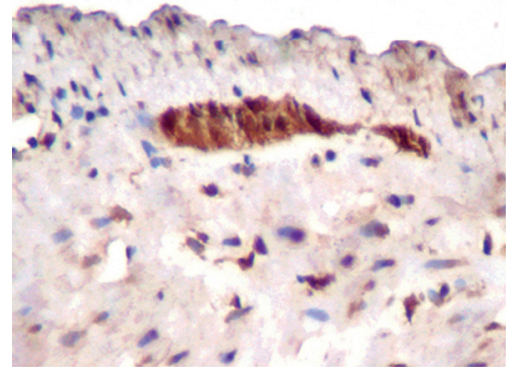

Normal group

(a)

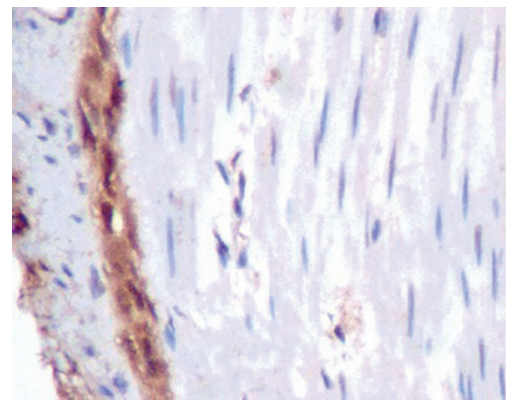

Normal group

(d)

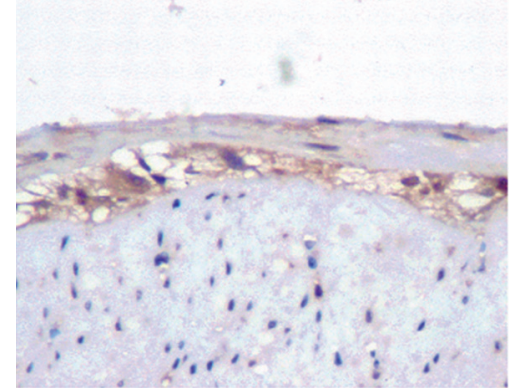

Model control group

(b)

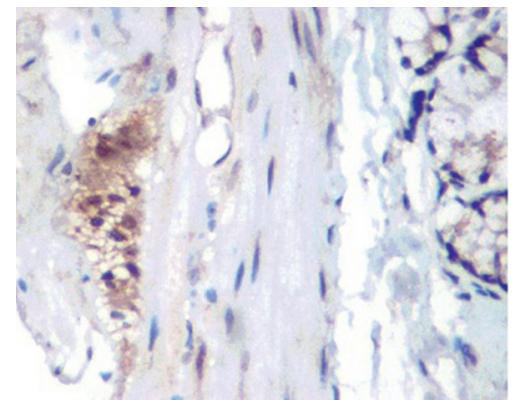

Model control group

(e)

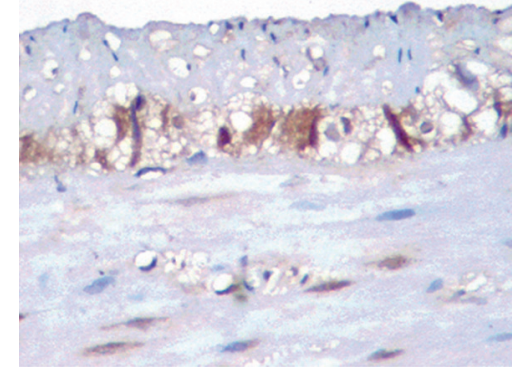

PEG-treated group

(c)

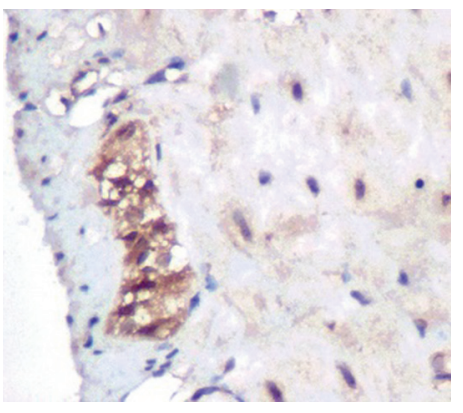

PEG-treated group

(f)

FIGURE 4: Immunocytochemical staining for expression of SCF (a-c) and c-kit (d-f) proteins in colonic tissues of rats after administration with or without PEG4,000 for $30 \mathrm{~d}(400 \times)$. PEG, polyethylene glycol; SCF, stem cell factor.

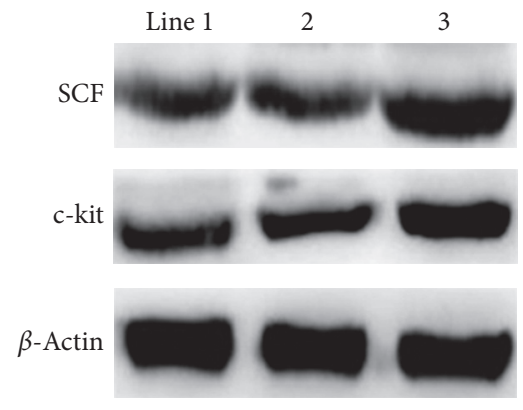

(a)

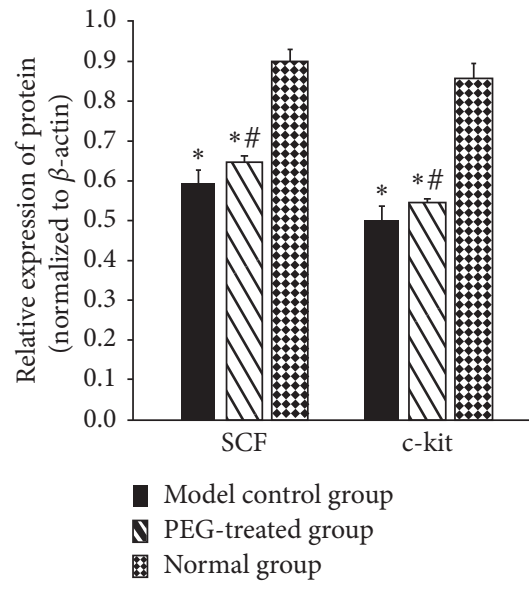

(b)

FIGURE 5: Expression level of SCF and c-kit proteins in colonic tissues of rats. (a) Constipated model rats were administered with either normal saline or PEG4,000 for 30d and then determined by western blot assay. Images shown are representative profiles from three technical replicates. Lines 1, 2, and 3 represent for model control group, PEG-treated group, and normal group, respectively. (b) Relative expression level of proteins in colonic tissues of rats. The data obtained from three independent experimental studies are presented as mean \pm standard deviation. If the error bar is not present, it is smaller than the thickness of graph line. ${ }^{*} P<0.05$, compared with normal group; ${ }^{\#} P>0.05$, compared with model control group. PEG, polyethylene glycol; SCF, stem cell factor.

suggest that long-term stimulation of anthraquinone laxatives can successfully induce constipation.

Mild constipation may be managed with dietary and lifestyle changes, whereas more patients with chronic constipation will require a range of treatments, which may include laxatives [31]. As a matter of fact, the regulation of gastrointestinal tract motility has been the main focus of constipation treatments, and the food intake and water 
consumption are considered to be important factors for evaluation of constipation symptoms and therapeutic effects [32]. PEG is recommended by the World's Gastrointestinal Organization in Constipation Guidelines (2010) [33], for it is not fermented by the gut flora and hence does not contribute to gas accumulation. Using the rhein-induced rat constipation model, the daily food intake and water consumption were maintained at constant levels compared with normal group. After intervention with PEG4,000 for $30 \mathrm{~d}$, fecal parameters including the frequency of defecation and fecal moisture were approximately similar to those in model control group. Of note, the first black time of stool discharge was not obviously shortened by PEG4,000 as compared with model control group, suggesting that PEG4,000 cannot effectively improve colon power or alleviate symptoms of constipation. Except for a significant reduction of fecal excretion, histological structure is considered to be one of the key markers of constipation in most studies [32, 34]. Some previously studies showed that thicknesses of villus layer, crypt layer, and muscle layer are regarded as important factors to evaluate the constipation symptoms and therapy effects of drugs [35, 36]. In our current study, morphological alterations including flatulence and dilation of the colon, accumulation of gas and fluid in intestinal cavity, and thinning of intestinal wall of colon were observed in model control group, and a similar result was observed in PEGtreated group. In addition, colonic sections showed an intact intestinal structure without any histological alterations in normal group, whereas chronic mucosal inflammation and vacuolar degeneration of myenteric plexus nerve cells were observed in model control group. Notably, administration of PEG4,000 at the selected dosages could not significantly restore abnormal structure of colon in constipated model rats.

It is well known the histopathology is an important clinical standard in the diagnosis of intestinal function. ICCs express the gene product of c-kit, a receptor tyrosine kinase, while SCF, the natural ligand for c-kit, is produced by smooth muscle cells [37]; thus the expression of SCF and c-kit proteins can be used to indicate alterations in ICCs throughout the gastrointestinal tracts in humans and other species. There are increasing researches regarding the importance of ICCs that are now well accepted to be the intestinal pacemaker cells for myogenic slow waves and phasic contractions of the gut wall, for they play an important role in regulating intestinal nerve signals to smooth muscle cells [38]. Recently, it has also been proved that decrease of ICCs in colonic tissues and structural damage can lead to a decrease of colon pacemaker function and attenuation of electrical excitation transmission, irregular slow wave resulting from smooth muscle disorder, contraction movement disorders, peristalsis decrease, or ineffective advance contractions $[39,40]$. In the current study, the expression of SCF was observed in all intestinal wall layers and deep brown granules appeared at the full-thickness of knot bowel wall in normal group, whereas that decreased significantly in model control group and only mild expression was shown as compared with model control group. Similarly, expression of c-kit was observed in the entire colon muscle layer, especially in the circular muscular layer and intramuscular plexus area, and deep brown granule could be seen on ICCs in normal group, while those in intramuscular plexus and ICCs appeared to be pale dyeing in model control group, suggesting that expression of SCF and c-kit decreased significantly in constipation model group compared with normal group. Of note, a similar trend of alteration was observed in PEG-treated group, but there was no significant difference compared with model control group. Collectively, all these results demonstrate that administration of PEG4,000 has no obviously protective effects on the colonic function.

Since there are no diagnostic histological features that can help determine its underlying aetiology, more and more researchers pay more attention to the SCF/c-kit signaling pathway closely related to the ICCs phenotype and function $[7,41]$. According to our present study, the expression level of SCF and c-kit was lower in colonic tissues of model control group as compared with normal group. After administration with PEG4,000, the level of SCF and c-kit proteins increased slightly in colonic tissues and the data failed to reach a statistical significance as compared with model control group. Even so, it is apparent that the signaling pathway of SCF/c-kit is at least partly involved in the rhein-induced SD rat constipations. A previously study on animals with constipation found that the expression level of SCF and c-kit declined with lower amounts of ICCs in colonic tissues [42]. Overall, it is supposed that constant stimulation of rhein can cause a decreased expression of SCF and c-kit in colonic tissues, and destroy the normal signaling pathways of SCF/c-kit and induce abnormal alternations of ICCs, consequently leading to a decrease of colon power. Nevertheless, it should be noted that there are some limits and restrictions to the clinical translation of results obtained from laxative-induced rats to human conditions.

In conclusion, although there is no perfect animal model that exhibits anatomical and functional defects consistent with constipation, the data presented here constitute strong evidence that long-term stimulation of anthraquinone rhein can cause a decrease of colon power and induction of constipation by inhibiting the normal SCF/c-kit signaling pathway, yet all these alterations cannot be relieved or restored by PEG4,000. All these findings strongly suggest that long-term use of anthraquinone laxatives should be avoided for clinical treatment of constipation.

\section{Data Availability}

The datasets used and analyzed in the present study are available from the corresponding author.

\section{Conflicts of Interest}

The authors declare that they have no conflicts of interest.

\section{Authors' Contributions}

Zhang B substantially acquired the funding, provided the conception, designed the research, and drafted the 
manuscript. Huo MD supervised the research and revised the manuscript. Chen ZC collected, analyzed, and interpreted the data. Gao F conducted the whole experiment. Liu $\mathrm{Y}$ and Zhou XX carried out the experiments. All authors read and approved the final manuscript.

\section{Acknowledgments}

This study was supported by the Fund for Project of Science and Technology of Jiangsu Province Chinese Medicine Bureau (PY201517) and National Science and Technology Support Program (2015BAI04B02).

\section{References}

[1] J. E. Kim, Y. J. Lee, M. H. Kwak, J. Ko, J. T. Hong, and D. Y. Hwang, "Aqueous extracts of Liriope platyphylla induced significant laxative effects on loperamide-induced constipation of SD rats," BMC Complementary and Alternative Medicine, vol. 13, p. 333, 2013.

[2] H. Chu and X. Hou, "Understanding of constipation symptoms and the diagnosis and management of constipation in Chinese physicians," PLoS One, vol. 11, Article ID e0152801, 2016.

[3] L. M. Herrick, W. M. Spalding, Y. A. Saito, J. Moriarty, and C. Schleck, "A case-control comparison of direct healthcareprovider medical costs of chronic idiopathic constipation and irritable bowel syndrome with constipation in a communitybased cohort," Journal of Medical Economics, vol. 20, no. 3, pp. 273-279, 2017.

[4] C. H. Huang, J. S. Lin, T. C. Li et al., "Comparison of a Chinese herbal medicine $(\mathrm{CCH} 1)$ and lactulose as first-line treatment of constipation in long-term care: a randomized, doubleblind, double-dummy, and placebo-controlled trial," Evidence-Based Complementary and Alternative Medicine, vol. 2012, Article ID 923190, 12 pages, 2012.

[5] H. Sun, G. Luo, D. Chen, and Z. Xiang, "A comprehensive and system review for the pharmacological mechanism of action of rhein, an active anthraquinone ingredient," Front Pharmacol, vol. 7, p. 247, 2016.

[6] W. Meng, J. Zhou, R. Elliott, P. Murphy, V. Ho, and M. O'Connor, "Is there a role for human pluripotent stem cells in modelling interstitial cells of Cajal and gut motility disorders?" Current Stem Cell Research \& Therapy, vol. 10, no. 3, pp. 251-257, 2015.

[7] Y. Chai, Y. Huang, H. Tang et al., "Role of stem cell growth factor/c-Kit in the pathogenesis of irritable bowel syndrome," Experimental and Therapeutic Medicine, vol. 13, no. 4, pp. 1187-1193, 2017.

[8] F. Zhu, S. Xu, Y. Zhang, F. Chen, J. Ji, and G. Xie, "Total glucosides of paeony promote intestinal motility in slow transit constipation rats through amelioration of interstitial cells of Cajal," PLoS One, vol. 11, Article ID e0160398, 2016.

[9] J. Zhou, M. D. O'Connor, and V. Ho, “The potential for gut organoid derived interstitial cells of Cajal in replacement therapy," International Journal of Molecular Sciences, vol. 18, Article ID e2059, 2017.

[10] M. Mínguez, A. López Higueras, and J. Júdez, "Use of polyethylene glycol in functional constipation and fecal impaction," Revista espanola de enfermedades digestivas: organo oficial de la Sociedad Espanola de Patologia Digestiva, vol. 108, no. 12, pp. 790-806, 2016.
[11] M. R. Esmaeilidooki, S. A. Mozaffarpur, M. Mirzapour, H. Shirafkan, M. Kamalinejad, and A. Bijani, "Comparison between the cassia fistula's emulsion with polyethylene glycol (PEG4000) in the pediatric functional constipation: a randomized clinical trial," Iranian Red Crescent Medical Journal, vol. 18, Article ID e33998, 2016.

[12] P. Chassagne, P. Ducrotte, P. Garnier, and H. MathiexFortunet, "Tolerance and long-term efficacy of polyethylene glycol 4000 (Forlax) compared to lactulose in elderly patients with chronic constipation," The Journal of Nutrition, Health \& Aging, vol. 21, no. 4, pp. 429-439, 2017.

[13] G. Li, Q. Wang, Y. Qian, Y. Zhou, R. Wang, and X. Zhao, "Component analysis of $\mathrm{Pu}$-erh and its anti-constipation effects," Molecular Medicine Reports, vol. 9, no. 5, pp. 2003-2009, 2014.

[14] K. Krogh, G. Chiarioni, and W. Whitehead, "Management of chronic constipation in adults," United European Gastroenterology Journal, vol. 5, no. 4, pp. 465-472, 2017.

[15] P. Enck, J. Leinert, M. Smid, T. Köhler, and J. SchwilleKiuntke, "Functional constipation and constipation-predominant irritable bowel syndrome in the general population: data from the GECCO study," Gastroenterology Research and Practice, vol. 2016, Article ID 3186016, 9 pages, 2016.

[16] J. Wang, J. H. Huang, Y. F. Cheng, and G. M. Yang, "Banana resistant starch and its effects on constipation model mice," Journal of Medicinal Food, vol. 17, no. 8, pp. 902-907, 2014.

[17] C. Liang, K.-Y. Wang, Z. Yu, and B. Xu, "Development of a novel mouse constipation model," World Journal of Gastroenterology, vol. 22, no. 9, pp. 2799-2810, 2016.

[18] P. Sun, Y. Zhou, R. Wang, and X. Zhao, "Preventive effect of Dendrobium candidum wall. ex Lindl. on activated carboninduced constipation in mice," Experimental and Therapeutic Medicine, vol. 9, pp. 563-568, 2015.

[19] G. Farrugia, S. Lei, X. Lin et al., "A major role for carbon monoxide as an endogenous hyperpolarizing factor in the gastrointestinal tract," Proceedings of the National Academy of Sciences, vol. 100, no. 14, pp. 8567-8570, 2003.

[20] F. Neri, G. Cavallari, M. Tsivian et al., "Effect of colic vein ligature in rats with loperamide-induced constipation," Journal of Biomedicine \& Biotechnology, vol. 2012, Article ID 896162, 5 pages, 2012.

[21] S. H. Han, K. Park, E. Y. Kim, S. H. Ahn, H. S. Lee, and H. J. Suh, "Cactus (Opuntia humifusa) water extract ameliorates loperamide-induced constipation in rats," vol. 17, p. 49, 2017.

[22] J. E. Kim, J. Go, E. K. Koh et al., "Gallotannin-enriched extract isolated from Galla Rhois may be a functional candidate with laxative effects for treatment of loperamide-induced constipation of SD rats," PLoS One, vol. 11, Article ID e0161144, 2016.

[23] P. Li, Q. Lu, W. Jiang et al., "Pharmacokinetics and pharmacodynamics of rhubarb anthraquinones extract in normal and disease rats," Biomedicine \& Pharmacotherapy, vol. 91, pp. 425-435, 2017.

[24] T. Bu, C. Wang, Q. Meng et al., "Hepatoprotective effect of rhein against methotrexate-induced liver toxicity," European Journal of Pharmacology, vol. 834, pp. 266-273, 2018.

[25] Z. Meng, Y. Yan, Z. Tang et al., "Anti-hyperuricemic and nephroprotective effects of rhein in hyperuricemic mice," Planta Medica, vol. 81, no. 4, pp. 279-285, 2015.

[26] H. Ge, H. Tang, Y. Liang et al., "Rhein attenuates inflammation through inhibition of NF- $\kappa$ B and NALP3 inflammasome in vivo and in vitro," Drug Design, Development and Therapy, vol. 11, pp. 1663-1671, 2017. 
[27] Q. Zhao, X. Wang, A. Chen et al., "Rhein protects against cerebral ischemic-/reperfusion-induced oxidative stress and apoptosis in rats," International Journal of Molecular Medicine, vol. 41, pp. 2802-2812, 2018.

[28] H. Suo, X. Zhao, Y. Qian et al., "Therapeutic effect of activated carbon-induced constipation mice with lactobacillus fermentum suo on treatment," International Journal of Molecular Sciences, vol. 15, no. 12, pp. 21875-21895, 2014.

[29] G. Daniels and M. Schmelzer, "Giving laxatives safely and effectively," MEDSURG Nursing, vol. 22, pp. 290-296, 2013.

[30] M. Y. Zhou, P. Jia, J. P. Chen et al., "Laxative effects of Salecan on normal and two models of experimental constipated mice," BMC Gastroenterology, vol. 13, p. 52, 2013.

[31] B. Serrano-Falcón and E. Rey, "The safety of available treatments for chronic constipation," Expert Opinion on Drug Safety, vol. 16, no. 11, pp. 1243-1253, 2017.

[32] J.-E. Kim, Y.-J. Lee, M.-H. Kwak et al., "Metabolomics approach to serum biomarker for loperamide-induced constipation in SD rats," Laboratory Animal Research, vol. 30, no. 1, pp. 35-43, 2014.

[33] H. Lee-Robichaud, K. Thomas, J. Morgan, and R. L. Nelson, "Lactulose versus polyethylene glycol for chronic constipation," Cochrane Database of Systematic Reviews, vol. 7, Article ID CD007570, 2010.

[34] J. E. Kim, J. Go, H. S. Lee, J. T. Hong, and D. Y. Hwang, "Spicatoside A in red liriope platyphylla displays a laxative effect in a constipation rat model via regulating $\mathrm{mAChRs}$ and ER stress signaling," International Journal of Molecular Medicine, vol. 43, no. 1, pp. 185-198, 2019.

[35] J. E. Kim, J. Go, J. E. Sung et al., "Uridine stimulate laxative effect in the loperamide induced constipation of SD rats through regulation of the mAChRs signaling pathway and mucin secretion," BMC Gastroenterol, vol. 17, p. 21, 2017.

[36] X. Zha, D. Lin, Y. Zhao, and X. Yang, "Bacterial cellulose relieves diphenoxylate-induced constipation in rats," Journal of Agricultural and Food Chemistry, vol. 66, pp. 4106-4117, 2018.

[37] F. Mei, J. Han, Y. Huang, Z.-Y. Jiang, C.-J. Xiong, and D.-S. Zhou, "Plasticity of interstitial cells of cajal: a study in the small intestine of adult Guinea pigs," The Anatomical Record: Advances in Integrative Anatomy and Evolutionary Biology, vol. 292, no. 7, pp. 985-993, 2009.

[38] G. Farrugia, "Interstitial cells of Cajal in health and disease," Neurogastroenterology \& Motility, vol. 20, pp. 54-63, 2008.

[39] B. Lies, K. Beck, J. Keppler, D. Saur, D. Groneberg, and A. Friebe, "Nitrergic signalling via interstitial cells of Cajal regulates motor activity in murine colon," The Journal of Physiology, vol. 593, no. 20, pp. 4589-4601, 2015.

[40] S. O. Kiriukhin and O. V. Makarova, "Morphological changes in the colonic muscular layer and interstitial cells of Cajal in experimental acute ulcerative colitis," Arkhiv Patologii, vol. 78, no. 5, pp. 27-32, 2016.

[41] Y. Y. Tan, Z. L. Ji, G. Zhao, J. R. Jiang, D. Wang, and J. M. Wang, "Decreased SCF/c-kit signaling pathway contributes to loss of interstitial cells of Cajal in gallstone disease," International Journal of Clinical and Experimental Medicine, vol. 7, no. 11, pp. 4099-4106, 2014.

[42] A. F. Brading and K. D. McCloskey, "Mechanisms of Disease: specialized interstitial cells of the urinary tract-an assessment of current knowledge," Nature Clinical Practice Urology, vol. 2, no. 11, pp. 546-554, 2005. 